\title{
Sidon sets and graphs without 4-cycles
}

\author{
Michael Tait* and Craig Timmons*
}

\begin{abstract}
The problem of determining the maximum number of edges in an $n$-vertex graph that does not contain a 4 -cycle has a rich history in extremal graph theory. Using Sidon sets constructed by Bose and Chowla, for each odd prime power $q$ we construct a graph with $q^{2}-q-2$ vertices that does not contain a 4 -cycle and has at least $\frac{1}{2} q^{3}-q^{2}-O\left(q^{3 / 4}\right)$ edges. This disproves a conjecture of Abreu, Balbuena, and Labbate concerning the Turán number ex $\left(q^{2}-q-\right.$ $\left.2, C_{4}\right)$.
\end{abstract}

KEYWORdS AND PHRASES: Turan number, Sidon set, 4-cycle.

\section{Introduction}

Let $F$ be a graph. The Turán number of $F$, denoted ex $(n, F)$, is the maximum number of edges in an $n$-vertex graph that does not contain $F$ as a subgraph. Determining ex $(n, F)$ for different graphs $F$ is one of the central problems in extremal combinatorics. One of the most studied cases is the Turán number of $C_{4}$, the cycle on four vertices. It is known that ex $\left(n, C_{4}\right) \leq$ $\frac{1}{2} n^{3 / 2}+o\left(n^{3 / 2}\right)$ for every $n \rightarrow \infty$ (see [2]). It is more difficult to construct $n$-vertex graphs without 4 -cycles that have $\frac{1}{2} n^{3 / 2}+o\left(n^{3 / 2}\right)$ edges. Using polarity graphs of projective planes, Brown [4], Erdős, Rényi, and Sós [7] independently proved that for each prime power $q, \operatorname{ex}\left(q^{2}+q+1, C_{4}\right) \geq \frac{1}{2} q(q+1)^{2}$. To define polarity graphs we need some terminology from finite geometry.

Let $\mathcal{P}$ and $\mathcal{L}$ be disjoint sets and $\mathcal{I} \subset \mathcal{P} \times \mathcal{L}$. Elements of $\mathcal{P}$ are called points, elements of $\mathcal{L}$ are called lines, and $\mathcal{I}$ defines an incidence relation on the pair $(\mathcal{P}, \mathcal{L})$. Let $\pi: \mathcal{P} \cup \mathcal{L} \rightarrow \mathcal{P} \cup \mathcal{L}$ be a bijection such that $\pi(\mathcal{P})=\mathcal{L}$, $\pi(\mathcal{L})=\mathcal{P}, \pi^{2}=\mathrm{id}$, and for all $p \in \mathcal{P}$ and $l \in \mathcal{L}$ we have $(p, l) \in \mathcal{I}$ if and only if $(\pi(l), \pi(p)) \in \mathcal{I}$. The map $\pi$ is a polarity of the geometry $(\mathcal{P}, \mathcal{L}, \mathcal{I})$. The polarity graph $G_{\pi}$ of the geometry $(\mathcal{P}, \mathcal{L}, \mathcal{I})$ with respect to $\pi$ is the graph with vertex set $V\left(G_{\pi}\right)=\mathcal{P}$ and edge set

$$
E\left(G_{\pi}\right)=\{\{p, q\}: p, q \in \mathcal{P}, p \neq q, \text { and }(p, \pi(q)) \in \mathcal{I}\} .
$$

arXiv: 1309.6350

*Both authors were partially supported by NSF Grant DMS-1101489 through Jacques Verstraëte. 
A point $p$ is an absolute point of $\pi$ if $(p, \pi(p)) \in \mathcal{I}$.

If $(\mathcal{P}, \mathcal{L}, \mathcal{I})$ is a finite projective plane of order $q$ and $\pi$ is an orthogonal polarity (one with exactly $q+1$ absolute points), then the polarity graph will have $q^{2}+q+1$ vertices, have $\frac{1}{2} q(q+1)^{2}$ edges, and will not contain a 4 -cycle. The constructions of [4] and [7] are polarity graphs of the projective plane $P G\left(2, \mathbb{F}_{q}\right)$ where $q$ is a prime power and $\mathbb{F}_{q}$ is the finite field with $q$ elements. The polarity is the orthogonal polarity sending the point $\left(x_{0}, x_{1}, x_{2}\right)$ to the line $\left[x_{0}, x_{1}, x_{2}\right]$ and vice versa (see [2] or [11] for more details). These polarity graphs show that for any prime power $q$, $\operatorname{ex}\left(q^{2}+q+1, C_{4}\right) \geq \frac{1}{2} q(q+1)^{2}$.

The exact value of $\operatorname{ex}\left(n, C_{4}\right)$ was determined using computer searches ([6], [14]) for all $n \leq 31$. Füredi [10] proved that whenever $q \geq 13$ is a prime power, $\operatorname{ex}\left(q^{2}+q+1, C_{4}\right) \leq \frac{1}{2} q(q+1)^{2}$ thus we get the exact result $\operatorname{ex}\left(q^{2}+q+1, C_{4}\right)=\frac{1}{2} q(q+1)^{2}$ for all prime powers $q \geq 13$. It was also shown in [10] that the only graphs with $q^{2}+q+1$ vertices and $\frac{1}{2} q(q+1)^{2}$ edges that do not contain 4-cycles are orthogonal polarity graphs of finite projective planes. Along with the constructions of [4] and [7], the results of Füredi are the most important contributions to the 4-cycle Turán problem. Recently Firke, Kosek, Nash, and Williford [9] proved that for even $q, \operatorname{ex}\left(q^{2}+\right.$ $\left.q, C_{4}\right) \leq \frac{1}{2} q(q+1)^{2}-q$. If $q$ is a power of two then we have the exact result $\operatorname{ex}\left(q^{2}+q, C_{4}\right)=\frac{1}{2} q(q+1)^{2}-q$. The lower bound in this case comes from taking an orthogonal polarity graph of a projective plane of order $q$ and removing a vertex of degree $q$.

The results we have mentioned so far describe all of the cases in which an exact formula for $\operatorname{ex}\left(n, C_{4}\right)$ is known. Using known results on densities of primes, one has the asymptotic result $\operatorname{ex}\left(n, C_{4}\right)=\frac{1}{2} n^{3 / 2}+o\left(n^{3 / 2}\right)$ but there are still many open problems concerning graphs with 4-cycles. For example, Erdös and Simonovits [8] conjectured that if $G$ is any $n$-vertex graph with $\operatorname{ex}\left(n, C_{4}\right)+1$ edges, then $G$ must contain at least $n^{1 / 2}+o\left(n^{1 / 2}\right)$ copies of $C_{4}$. For more on the Turán problems for $C_{4}$ and other bipartite Turán problems we refer the reader to the excellent survey of Füredi and Simonovits [11].

While investigating adjacency matrices of polarity graphs of $P G\left(2, \mathbb{F}_{q}\right)$ with respect to the orthogonal polarity, Abreu, Balbuena, and Labbate [1] were able to find subgraphs of a polarity graph that have many edges. By deleting such a subgraph, Abreu et al. [1] proved that for any prime power $q$,

$$
\operatorname{ex}\left(q^{2}-q-2, C_{4}\right) \geq \begin{cases}\frac{1}{2} q^{3}-q^{2}-\frac{q}{2}+1 & \text { if } q \text { is odd } \\ \frac{1}{2} q^{3}-q^{2} & \text { if } q \text { is even. }\end{cases}
$$

They conjectured that these bounds are best possible. Our main result shows that when $q$ is an odd prime power, this lower bound can be improved by $\frac{q}{2}-O\left(q^{3 / 4}\right)$. 
Theorem 1.1. If $q$ is an odd prime power, then

$$
\operatorname{ex}\left(q^{2}-q-2, C_{4}\right) \geq \frac{1}{2} q^{3}-q^{2}-O\left(q^{3 / 4}\right) .
$$

We will construct graphs without 4-cycles using the Sidon sets constructed by Bose and Chowla [3]. Let $\Gamma$ be an abelian group. A set $A \subset \Gamma$ is a Sidon set if whenever $a+b=c+d$ with $a, b, c, d \in A$, the pair $(a, b)$ is a permutation of $(c, d)$. Sidon sets are well studied objects in combinatorial number theory and for more on Sidon sets we recommend O'Bryant's survey [13].

Let $q$ be a prime power and $\theta$ be a generator of the multiplicative group $\mathbb{F}_{q^{2}}^{*}$ where $\mathbb{F}_{q^{2}}^{*}$ is the nonzero elements of the finite field $\mathbb{F}_{q^{2}}$. Bose and Chowla proved [3] that

$$
A(q, \theta):=\left\{a \in \mathbb{Z}_{q^{2}-1}: \theta^{a}-\theta \in \mathbb{F}_{q}\right\}
$$

is a Sidon set in the group $\mathbb{Z}_{q^{2}-1}$. Furthermore,

$$
|A(q, \theta)|=q .
$$

To see this, one observes that $\theta^{a}-\theta=\theta^{b}-\theta$ implies that $a \equiv b\left(\bmod q^{2}-1\right)$. In addition, since $\theta$ generates $\mathbb{F}_{q^{2}}^{*}, \mathbb{F}_{q}^{*} \subset \mathbb{F}_{q^{2}}^{*}=\left\{\theta^{a}: a \in \mathbb{Z}_{q^{2}-1}\right\}=\left\{\theta^{a}-\theta\right.$ : $\left.a \in \mathbb{Z}_{q^{2}-1}\right\}$.

Definition 1.2. Let $q$ be a prime power and $\theta$ be a generator of the multiplicative group $\mathbb{F}_{q^{2}}^{*}$. The graph $G_{q, \theta}$ is the graph with vertex set $\mathbb{Z}_{q^{2}-1}$ and two distinct vertices $i$ and $j$ are adjacent if and only if $i+j=a$ for some $a \in A(q, \theta)$.

It is known that Sidon sets can be used to construct graphs without 4-cycles. We will prove a result about the Bose-Chowla Sidon sets (see Lemma 2.6) that helps us find a subgraph of $G_{q, \theta}$ with $q+1$ vertices that contains many edges. We remove this subgraph to obtain a graph with $q^{2}-q-2$ vertices and at least $\frac{1}{2} q^{3}-q^{2}-O\left(q^{3 / 4}\right)$ edges. In addition to providing examples of graphs with no 4 -cycles, the graphs $G_{q, \theta}$ have been used to solve other extremal problems (see [5]).

We would like to remark that we could have defined $G_{q, \theta}$ as a polarity graph in the following way. Let $\mathcal{P}=\mathbb{Z}_{q^{2}-1}$ and let $\mathcal{L}$ be the set of $q^{2}-1$ translates of $A(q, \theta)$. That is, $\mathcal{L}=\left\{A_{1}, A_{2}, \ldots, A_{q^{2}-1}\right\}$ where $A_{i}:=A(q, \theta)+$ $i$. This defines a geometry in the obvious way; $i \in \mathcal{P}$ is incident to $A_{j} \in \mathcal{L}$ if and only if $i \in A_{j}$. We define a polarity by $\pi(i)=A_{q^{2}-1-i}$ for all $i \in \mathcal{P}$, and $\pi\left(A_{i}\right)=q^{2}-1-i$ for all $A_{i} \in \mathcal{L}$. The fact that $\pi$ is a polarity can be checked directly. We choose to use Definition 1.2 as it is more convenient for our argument. 


\section{Proof of Theorem 1.1}

In this section we fix an odd prime power $q$ and a generator $\theta$ of the multiplicative group $\mathbb{F}_{q^{2}}^{*}$. We write $A$ for the Sidon set $A(q, \theta)$ in $\mathbb{Z}_{q^{2}-1}$ and observe that $|A|=q$ by (1). All of our manipulations will be done in the group $\mathbb{Z}_{q^{2}-1}$ or in the finite field $\mathbb{F}_{q^{2}}$. If it is not clear from the context, we will state which algebraic structure we are working in.

The first two lemmas are known. We present proofs for completeness.

Lemma 2.1. The graph $G_{q, \theta}$ does not contain a 4-cycle.

Proof. Suppose $i j k l$ is a 4 -cycle in $G_{q, \theta}$. There are elements $a, b, c, d \in A$ such that $i+j=a, j+k=b, k+l=c$, and $l+i=d$. This implies

$$
a+c=b+d
$$

Since $A$ is a Sidon set, $(a, c)$ is a permutation of $(b, d)$. If $a=b$ then $i+j=$ $j+k$ so $i=k$. If $a=d$ then $i+j=l+i$ so $j=l$. In either case we have a contradiction thus $G_{q, \theta}$ does not contain a 4 -cycle.

Lemma 2.2. If $A-A:=\{a-b: a, b \in A\}$ then

$$
A-A=\mathbb{Z}_{q^{2}-1} \backslash\{q+1,2(q+1), 3(q+1), \ldots,(q-2)(q+1)\} .
$$

Proof. Suppose $s(q+1) \in A-A$ for some $1 \leq s \leq q-2$. Write $s(q+1)=a-b$ where $a, b \in A$ and $a \neq b$. We have for some $\alpha, \beta \in \mathbb{F}_{q}$,

$$
\theta^{s(q+1)}=\theta^{a-b}=\theta^{a} \theta^{-b}=(\theta+\alpha)(\theta+\beta)^{-1} .
$$

From this we obtain

$$
\theta+\alpha=(\theta+\beta)\left(\theta^{q+1}\right)^{s}
$$

but $\theta^{q+1} \in \mathbb{F}_{q}$ so $\theta+\alpha=(\theta+\beta) \gamma$ for some $\gamma \in \mathbb{F}_{q}$. Since $\theta$ does not satisfy a nontrivial linear relation over $\mathbb{F}_{q}$ we must have $\gamma=1$ hence $\alpha=\beta$ (in $\mathbb{F}_{q^{2}}$ ) so $a=b\left(\right.$ in $\left.\mathbb{Z}_{q^{2}-1}\right)$. From this we get $s(q+1)=0$ which contradicts the fact that $1 \leq s \leq q-2$. This shows that

$$
(A-A) \cap\{q+1,2(q+1), \ldots,(q-2)(q+1)\}=\emptyset .
$$

Since $A$ is a Sidon set, $|A-A|=q(q-1)+1$ which is precisely the number of elements in the set

$$
\mathbb{Z}_{q^{2}-1} \backslash\{q+1,2(q+1), \ldots,(q-2)(q+1)\}
$$

and this completes the proof of the lemma. 
We note that for $i$ a vertex in $G_{q, \theta}$, if $i$ is an absolute point (see the end of Section 1), then $i+i \in A$ and the degree of $i$ is $q-1$. If $i$ is not an absolute point, then $i+i \notin A$ and the degree of $i$ is $q$.

Lemma 2.3. Distinct vertices $i$ and $j$ in $G_{q, \theta}$ have a common neighbor if and only if $i-j \in(A-A) \backslash\{0\}$.

Proof. First suppose $i$ and $j$ are distinct vertices that have a common neighbor $k$. Then $i+k=a$ and $k+j=b$ for some $a, b \in A$ so $i-j=$ $(a-k)-(b-k)=a-b$. Since $i \neq j$, we get that $a-b \neq 0$.

Now suppose $i-j=a-b$ for some $a, b \in A$ with $a \neq b$. Let $k=a-i$. Then $k+i=a$ so $k$ is adjacent to $i$. Also, $k=a-i=b-j$ so $k+j=b$ and $k$ is adjacent to $j$.

Lemma 2.4. If $i$ is an absolute point then $i+\frac{q^{2}-1}{2}$ is also an absolute point. Proof. If $2 i=a$ for some $a \in A$ then $2\left(i+\frac{q^{2}-1}{2}\right)=2 i=a$.

Lemma 2.5. Let $i$ and $j$ be two distinct absolute points of $G_{q, \theta}$. If $i \neq$ $j+\frac{q^{2}-1}{2}$ then $i$ and $j$ have a common neighbor and if $i=j+\frac{q^{2}-1}{2}$ then $i$ and $j$ do not have a common neighbor.

Proof. By Lemmas 2.2 and 2.3, $i$ and $j$ have a common neighbor unless $i-j=s(q+1)$ for some $1 \leq s \leq q-2$. Since $i$ and $j$ are absolute points, there exist elements $a, b \in A$ such that $2 i=a$ and $2 j=b$ thus $a-b=2 s(q+1)$. By Lemma 2.2, it must be the case that $a=b$ so $2 i=2 j$. The solutions to $2 x \equiv 2 y\left(\bmod q^{2}-1\right)$ are $x=y$ and $x=y+\frac{q^{2}-1}{2}$ hence $i=j$ or $i=j+\frac{q^{2}-1}{2}$. Thus $i$ and $j$ will have a common neighbor whenever they are distinct absolute points with $i \neq j+\frac{q^{2}-1}{2}$ and will not have a common neighbor when $i=j+\frac{q^{2}-1}{2}$.

Lemma 2.6. Let $\left\{a_{1}, a_{2}, a_{3}\right\}$ and $\left\{b_{1}, b_{2}, b_{3}\right\}$ be subsets of $A$ with $a_{1}, a_{2}$, and $a_{3}$ all distinct and $b_{1}, b_{2}$, and $b_{3}$ all distinct. If

$$
2 b_{1}-a_{1}=2 b_{2}-a_{2}=2 b_{3}-a_{3}
$$

then two of the ordered pairs $\left(a_{1}, b_{1}\right),\left(a_{2}, b_{2}\right),\left(a_{3}, b_{3}\right)$ are equal.

The proof of Lemma 2.6 is simple but it is not short. For this reason we postpone the proof until after the proof of Theorem 1.1.

Lemma 2.7. Any vertex $j$ is adjacent to at most two absolute points. 
Proof. Suppose $j$ is a vertex of $G_{q, \theta}$ that is adjacent to three distinct absolute points $i_{i}, i_{2}$, and $i_{3}$. There exist elements $a_{1}, a_{2}, a_{3}, b_{1}, b_{2}, b_{3} \in A$ such that

$$
2 i_{k}=a_{k} \text { and } i_{k}+j=b_{k}
$$

for $k=1,2,3$. Since $i_{1}, i_{2}, i_{3}$ are all distinct, $b_{1}, b_{2}$, and $b_{3}$ must all be distinct. If $a_{k}=a_{l}$ for some $1 \leq k<l \leq 3$, then $i_{k}=i_{l}+\frac{q^{2}-1}{2}$. In this case, the vertices $i_{k}$ and $i_{l}$ are absolute points with a common neighbor but this is impossible by Lemma 2.5. We conclude that $a_{1}, a_{2}$, and $a_{3}$ are all distinct. For each $k$, we can write $i_{k}+j=b_{k}$ as $2 j=2 b_{k}-a_{k}$ so that

$$
2 b_{1}-a_{2}=2 b_{2}-a_{2}=2 b_{3}-a_{3} .
$$

By Lemma 2.6, $\left(a_{k}, b_{k}\right)=\left(a_{l}, b_{l}\right)$ for some $1 \leq k<l \leq 3$ but we have already argued that $a_{k}$ and $a_{l}$ are distinct. This gives the needed contradiction and completes the proof of the lemma.

Proof of Theorem 1.1. Let $P$ be the absolute points of $G_{q, \theta}$. Lemma 2.4 implies that the absolute points come in pairs so we can write

$$
P=\left\{i_{1}, i_{1}+\frac{q^{2}-1}{2}, i_{2}, i_{2}+\frac{q^{2}-1}{2}, \ldots, i_{t}, i_{t}+\frac{q^{2}-1}{2}\right\}
$$

where $2 t$ is the number of absolute points of $G_{q, \theta}$. When $q$ is odd, $q^{2}-1$ is even and we can write $q^{2}-1=2^{r} m$ where $r \geq 1$ is an integer and $m$ is odd. If $a \in A$, then the congruence

$$
2 x \equiv a\left(\bmod 2^{r} m\right)
$$

has no solution when $a$ is odd and two solutions if $a$ is even. Therefore $t$ is exactly the number of even elements of $A$ when we view $A$ as a subset of $\mathbb{Z}$. Lindström [12] proved that dense Sidon sets are close to evenly distributed among residue classes. In particular, the results of [12] imply that

$$
t=\frac{q}{2}+O\left(q^{3 / 4}\right)
$$

so we know that we have $q+O\left(q^{3 / 4}\right)$ absolute points in $G_{q, \theta}$. The number of vertices of $G_{q, \theta}$ is $q^{2}-1$ and the number of edges of $G_{q, \theta}$ is

$$
e(G)=\frac{1}{2}\left(q\left(q^{2}-1-2 t\right)+(q-1)(2 t)\right)=\frac{1}{2} q^{3}-\frac{1}{2} q-t .
$$


Let $S \subset V\left(G_{q, \theta}\right)$ with $|S|=q+1$ and let $t_{S}$ be the number of absolute points in $S$. The graph $G_{q, \theta} \backslash S$ has $q^{2}-q-2$ vertices and

$$
\frac{1}{2} q^{3}-\frac{1}{2} q-t-e(S)-e(S, \bar{S})
$$

edges. Here $e(S, \bar{S})$ is the number of edges of $G_{q, \theta}$ with exactly one endpoint in $S$. We can rewrite $e(S)+e(S, \bar{S})$ as

$$
\begin{aligned}
e(S)+e(S, \bar{S})=\sum_{i \in S} d(i)-e(S) & =\left(q+1-t_{S}\right) q+t_{S}(q-1)-e(S) \\
& =q^{2}+q-t_{S}-e(S) .
\end{aligned}
$$

By (3) we can write the number of edges of $G_{q, \theta} \backslash S$ as

(4) $\frac{1}{2} q^{3}-\frac{1}{2} q-t-\left(q^{2}+q-t_{S}-e(S)\right)=\frac{1}{2} q^{3}-q^{2}-\frac{3}{2} q-t+t_{S}+e(S)$.

For any $1 \leq j_{1}<j_{2} \leq t$, the pair $i_{j_{1}}$ and $i_{j_{2}}$ of absolute points have a unique common neighbor by Lemmas 2.5 and 2.1. Set $k=\left\lfloor\frac{1}{2} \sqrt{8 q+9}-\frac{1}{2}\right\rfloor$ and note that for large enough $q$ we have $k \leq t$. The integer $k$ is chosen so that it is as large as possible and still satisfies the inequality $\left(\begin{array}{l}k \\ 2\end{array}\right)+k \leq q+1$. Let $S_{1}=\left\{i_{1}, \ldots, i_{k}\right\}$. For each pair $1 \leq j_{1}<j_{2} \leq k$, let $x_{j_{1}, j_{2}}$ be the unique common neighbor of the absolute points $i_{j_{1}}$ and $i_{j_{2}}$. Let $S_{2}=\left\{x_{j_{1}, j_{2}}: 1 \leq\right.$ $\left.j_{1}<j_{2} \leq k\right\}$. By Lemma 2.7, $S_{2}$ consists of $\left(\begin{array}{l}k \\ 2\end{array}\right)$ distinct vertices. A short calculation shows that $\left(\begin{array}{l}k \\ 2\end{array}\right)+k \geq q-O(\sqrt{q})$. Let $S_{3}$ be a set of $q+1-\left(\begin{array}{c}k \\ 2\end{array}\right)-k$ vertices chosen arbitrarily from $V\left(G_{q, \theta}\right) \backslash\left(S_{1} \cup S_{2}\right)$. Let $S$ be the subgraph of $G_{q, \theta}$ induced by the vertices $S_{1} \cup S_{2} \cup S_{3}$. By construction, $S$ has $q+1$ vertices and at least $2\left(\begin{array}{c}k \\ 2\end{array}\right)$ edges so

$$
t_{S}+e(S) \geq k+2\left(\begin{array}{l}
k \\
2
\end{array}\right) \geq 2 q-O(\sqrt{q}) .
$$

By (2) and (4), removing the vertices of $S$ from $G_{q, \theta}$ leaves a graph with $q^{2}-q-2$ vertices and at least

$$
\frac{1}{2} q^{3}-q^{2}-2 q+2 q-O\left(q^{3 / 4}\right)=\frac{1}{2} q^{3}-q^{2}-O\left(q^{3 / 4}\right)
$$

edges.

Now we return to the proof of Lemma 2.6. 
Proof of Lemma 2.6. Let $\left\{a_{1}, a_{2}, a_{3}\right\},\left\{b_{1}, b_{2}, b_{3}\right\} \subset A$ with $a_{1}, a_{2}$, and $a_{3}$ all distinct, and $b_{1}, b_{2}$, and $b_{3}$ all distinct. Since $a_{k}, b_{k} \in A$, there exist elements $c_{k}, d_{k} \in \mathbb{F}_{q}$ such that

$$
\theta^{a_{k}}=\theta+c_{k} \text { and } \theta^{b_{k}}=\theta+d_{k}
$$

for $k=1,2,3$. Observe that $c_{1}, c_{2}$, and $c_{3}$ are all distinct and so are $d_{1}, d_{2}$, and $d_{3}$.

The generator $\theta$ satisfies a degree two polynomial over $\mathbb{F}_{q}$, say $\theta^{2}=\alpha \theta+\beta$ where $\alpha, \beta \in \mathbb{F}_{q}$. Since $\theta$ generates $\mathbb{F}_{q^{2}}^{*}$, it cannot be the case that $\alpha=0$ and if $\beta=0$, then $\theta(\theta-\alpha)=0$ which is impossible since $\theta \notin \mathbb{F}_{q}$. The polynomial $X^{2}-3 X+3 \beta \in \mathbb{F}_{q}[X]$ has at most two roots in $\mathbb{F}_{q}$. Without loss of generality, we may assume that

$$
c_{1}^{2}-3 c_{1} \alpha+3 \beta \neq 0
$$

since $c_{1}, c_{2}$, and $c_{3}$ are all distinct. This fact will be important towards the end of the proof.

Consider the equation $2 b_{1}+a_{2}=2 b_{2}+a_{1}$. We can rewrite this as

$$
\left(\theta+d_{1}\right)^{2}\left(\theta+c_{2}\right)=\left(\theta+d_{2}\right)^{2}\left(\theta+c_{1}\right)
$$

If we expand, use $\theta^{2}=\alpha \theta+\beta$, and regroup we obtain

$$
\begin{aligned}
& \theta\left(2 d_{1} \alpha+c_{2} \alpha+d_{1}^{2}+2 d_{1} c_{2}\right)+\left(2 d_{1} \beta+c_{2} \beta+d_{1}^{2} c_{2}\right) \\
= & \theta\left(2 d_{2} \alpha+c_{1} \alpha+d_{2}^{2}+2 d_{2} c_{1}\right)+\left(2 d_{2} \beta+c_{1} \beta+d_{2}^{2} c_{1}\right) .
\end{aligned}
$$

These coefficients are all in $\mathbb{F}_{q}$ so we must have

$$
2 d_{1} \alpha+c_{2} \alpha+d_{1}^{2}+2 d_{1} c_{2}=2 d_{2} \alpha+c_{1} \alpha+d_{2}^{2}+2 d_{2} c_{1}
$$

and

$$
2 d_{1} \beta+c_{2} \beta+d_{1}^{2} c_{2}=2 d_{2} \beta+c_{1} \beta+d_{2}^{2} c_{1}
$$

Similar arguments show that both (6) and (7) hold with $c_{3}$ replacing $c_{2}$ and $d_{3}$ replacing $d_{2}$. We view $c_{1}$ and $d_{1}$ as begin fixed and $\left(c_{2}, d_{2}\right)$ and $\left(c_{3}, d_{3}\right)$ as solutions to the system

$$
2 d_{1} \alpha+X \alpha+d_{1}^{2}+2 d_{1} X=2 Y \alpha+c_{1} \alpha+Y^{2}+2 Y c_{1}
$$




$$
2 d_{1} \beta+X \beta+d_{1}^{2} X=2 Y \beta+c_{1} \beta+Y^{2} c_{1} .
$$

One solution is $(X, Y)=\left(c_{1}, d_{1}\right)$. If we can show that the system (8), (9) has at most two solutions then we are done as this forces two of the pairs $\left(c_{1}, d_{1}\right),\left(c_{2}, d_{2}\right),\left(c_{3}, d_{3}\right)$ to be the same and the pair $\left(c_{k}, d_{k}\right)$ uniquely determines the pair $\left(a_{k}, b_{k}\right)$. Multiply (8) by $c_{1}$ and then subtract (9) to eliminate $Y^{2}$ and obtain

$\left(2 c_{1} d_{1} \alpha+c_{1} d_{1}^{2}+c_{1} \beta-2 d_{1} \beta-c_{1}^{2} \alpha\right)+X\left(\alpha c_{1}+2 c_{1} d_{1}-\beta-d_{1}^{2}\right)=Y\left(2 c_{1}^{2}+2 c_{1} \alpha-2 \beta\right)$.

Next we subtract $\alpha$ times (9) from $\beta$ times (8) to get

$$
d_{1}^{2} \beta+X\left(2 d_{1} \beta-d_{1}^{2} \alpha\right)=Y^{2}\left(\beta-\alpha c_{1}\right)+Y\left(2 c_{1} \beta\right) .
$$

If we knew that the coefficient of $X$ was nonzero in (10) and $\beta-\alpha c_{1} \neq 0$ then we could easily deduce that there are at most two solutions $(X, Y)$. Unfortunately we do not know this and so we have to work to overcome this obstacle.

Suppose (10) is an equation where the coefficients of $X$ and $Y$ are both 0 . Then

$$
2 c_{1}^{2}+2 c_{1} \alpha-2 \beta=0 \text { and } \alpha c_{1}+2 c_{1} d_{1}-\beta-d_{1}^{2}=0 .
$$

Since $q$ is odd, the first equation can be rewritten as $c_{1}^{2}+c_{1} \alpha-\beta$. Subtracting the second equation $c_{1}^{2}+c_{1} \alpha-\beta$ gives $c_{1}^{2}-2 c_{1} d_{1}+d_{1}^{2}=0$ hence $\left(c_{1}-d_{1}\right)\left(c_{1}+\right.$ $\left.d_{1}\right)=0$.

If $c_{1}=d_{1}$ then $\theta^{a_{1}}=\theta+c_{1}=\theta+d_{1}=\theta^{b_{1}}$ so $a_{1}=b_{1}$ (in $\mathbb{Z}_{q^{2}-1}$ ). Using $2 b_{1}-a_{1}=2 b_{2}-a_{2}$ we get $b_{1}+a_{2}=b_{2}+b_{2}$ so $b_{1}=b_{2}$, a contradiction. Assume $c_{1}=-d_{1}$. Then $c_{1} \neq 0$ and $d_{1} \neq 0$ otherwise $c_{1}=d_{1}$ which we already know does not occur. Since both coefficients of $X$ and $Y$ are 0 in (10) the constant term must also be 0 so, using $c_{1}=-d_{1}$,

$$
\begin{aligned}
0 & =2 c_{1} d_{1} \alpha+c_{1} d_{1}^{2}+c_{1} \beta-2 d_{1} \beta-c_{1}^{2} \alpha \\
& =-3 c_{1}^{2} \alpha+c_{1}^{3}+3 c_{1} \beta \\
& =c_{1}\left(c_{1}^{2}-3 c_{1} \alpha+3 \beta\right) .
\end{aligned}
$$

By (5) this is impossible. We conclude that at least one of the coefficients of $X$ or $Y$ in (10) must be nonzero.

If the coefficient of $X$ in (10) is nonzero then we can write $X=\gamma_{1} Y+\gamma_{2}$ for some $\gamma_{1}, \gamma_{2} \in \mathbb{F}_{q}$. Substituting this equation into (8) gives a quadratic 
equation in $Y$ which has at most two solutions and $Y$ uniquely determines $X$ since $X=\gamma_{1} Y+\gamma_{2}$ and we are done.

Assume now that $\alpha c_{1}+2 c_{1} d_{1}-\beta-d_{1}^{2}=0$. Then (10) gives a unique solution for $Y$. Since $(X, Y)=\left(c_{1}, d_{1}\right)$ is a solution, we must have that all solutions to the system (8), (9) have $Y=d_{1}$. Substituting into (8) and (9) we get

$$
\begin{gathered}
X\left(\alpha+2 d_{1}\right)=c_{1}\left(\alpha+2 d_{1}\right) \\
X\left(\beta+d_{1}^{2}\right)=c_{1}\left(\beta+d_{1}^{2}\right) .
\end{gathered}
$$

If $d_{1}=0$ then $X \alpha=c_{1} \alpha$ and since $\alpha \neq 0$ we get $X=c_{1}$ are we are done.

Assume $d_{1} \neq 0$. If either $\alpha+2 d_{1}$ or $\beta+d_{1}^{2}$ are nonzero then we are done. Assume $\alpha+2 d_{1}=\beta+d_{1}^{2}=0$. If we substitute $Y=d_{1}$ into (11) then we get

$$
X d_{1}\left(2 \beta-d_{1} \alpha\right)=d_{1} c_{1}\left(2 \beta-d_{1} \alpha\right)
$$

Again, if $2 \beta-d_{1} \alpha$ is nonzero we are done, so assume $2 \beta-d_{1} \alpha=0$. Using the three equations

$$
\alpha+2 d_{1}=0, \beta+d_{1}^{2}=0,2 \beta-d_{1} \alpha=0
$$

we have

$$
0=2 \beta-d_{1} \alpha=2\left(-d_{1}^{2}\right)-d_{1}\left(-4 d_{1}\right)=2 d_{1}^{2}
$$

so $d_{1}=0$ giving the needed contradiction.

\section{References}

[1] M. Abreu, C. Balbuena, D. Labbate, Adjacency matrices of polarity graphs and other $C_{4}$-free graphs of large size, Des. Codes Cryptogr. (2010), 55, 221-233. MR2608429

[2] B. Bollobás, Extremal Graph Theory, Academic Press Inc. (London) Ltd., 1978. MR0506522

[3] R. C. Bose, S. Chowla, Theorems in the additive theory of numbers, Comment. Math. Helv. 37 (1962), 141-147. MR0144877

[4] W. G. Brown, On graphs that do not contain a Thomsen graph, Canada Math. Bull. 9 (1966), 281-289. MR0200182

[5] F. R. K. Chung, Diameters and eigenvalues, Journal of the American Mathematical Society, Vol. 2, No. 2, 187-196, 1989. MR0965008 
[6] C. R. J. Clapham, A. Flockhart, J. Sheehan, Graphs without four-cycles, J. Graph Theory 13 (1989), no. 1, 29-47. MR0982865

[7] P. Erdős, A. Rényi, V. T. Sós, On a problem of graph theory, Studia Sci. Math. Hungar. 1 (1966), 215-235. MR0223262

[8] P. Erdős, M. Simonovits, Cube-saturated graphs and related problems, Progress in Graph Theory (Waterloo, Ont., 1982), 203-218, Academic Press, Toronto, 1984. MR0776802

[9] F. Firke, P. Kosek, E. Nash, J. Williford, Extremal graphs without 4-cycles, Journal of Combinatorial Theory, Series B 103, 327-336 (2013). MR3048158

[10] Z. Füredi, On the number of edges of quadrilateral-free graphs, Journal of Combinatorial Theory, Series B 68, 1-6 (1996). MR1405701

[11] Z. Füredi, M. Simonovits, The history of degenerate (bipartite) extremal graph problems, arXiv:1306.5167.

[12] B. Lindström, Well distribution of Sidon sets in residue classes, Journal of Number Theory 69 (2), 197-200 (1998). MR1617317

[13] K. O'Bryant, A complete annotated bibliography of work related to Sidon sequences, Electronic J. of Combinatorics DS 11 (2004).

[14] P. Rowlinson, Y. Yuansheng, On extremal graphs without four-cycles, Utilitas Math. 41 (1992), 204-210. MR1162526

Michael TAit

Department of MATHEMATICS

University of California, San Diego

USA

E-mail address: mtait@math.ucsd.edu

Craig Timmons

Department of Mathematics

University of California, San Diego

USA

E-mail address: ctimmons@math.ucsd.edu

ReCeived September 25, 2013 